\title{
DIFFERENTIAL EXPRESSION OF LHCII GENES IN MESOPHYLL AND BUNDLE SHEATH CELLS OF MAIZE
}

\author{
by \\ ROBERTO BASSI ${ }^{1)}$ and DAVID J. SIMPSON \\ Department of Physiology, Carlsberg Laboratory, \\ Gl. Carlsbergvej 10, DK-2500 Copenhagen Valby \\ " Permanent address: Dipartimento di Biologia, Università di Padova, \\ via Orto Botanico 35, 35100 Padova, Italy
}

Keywords: Freeze-fracture, light-harvesting complex, photosystem I and II, polypeptide composition, protoplasts, stroma lamellae, thylakoids

\begin{abstract}
The properties and composition of bundle sheath and mesophyll thylakoids from maize leaves are compared. This was possible because of the isolation of large amounts of purified material obtained by enzymatic digestion of mechanically disrupted leaves. Bundle sheath thylakoids from mature leaves, lack the chlorophyll-proteins and polypeptides associated with the reaction centre of photosystem II. They do, however, contain significant amounts of LHCII, which transfers excitation energy to photosystem I. LHCII isolated from bundle sheath thylakoids had a different freeze-fracture ultrastructure and a different polypeptide composition from LHCII isolated from mesophyll thylakoids, indicating a differential expression of the LHCII gene family in mesophyll and bundle sheath cells of maize leaves.
\end{abstract}

\section{INTRODUCTION}

The photosynthetic membranes of higher plants are now thought to be characterised by extreme lateral heterogeneity with respect to composition and function, with all photosystem I (PSI) in the unstacked membrane regions (stroma làmellae) and most, if not all photosystem II (PSII) in the appressed regions (grana) (1). The locations of some of the minor components remain in doubt because the methods used for the isolation of stroma and grana lamellae requires either mechanical disruption or detergent fractionation. Both procedures can generate artefacts due to cross-contamination or selective removal of some components.

Chloroplasts containing no grana lamellae can therefore be very useful for studying thylakoid composition since their isolation avoids these procedures. Such chloroplasts are found in the bundle sheath cells (11) of some C4 plants (e.g., maize and sorghum) characterised by high levels of NADPH-malic enzyme. In the leaves of such plants, $\mathrm{CO}_{2}$ is fixed by phosphoenolpyruvate cärboxylase in mesophyll cells and transported as malate to bundle sheath cells, where decarboxylation yields $\mathrm{CO}_{2}$ and NADPH. The

\footnotetext{
Abbreviations: $\mathrm{BS}=$ bundle sheath; $\mathrm{Chl}=$ chlorophyll; $\mathrm{DCPIP}=2,6$-di-chlorophenol indophenol; $\mathrm{DPC}=$ 1,5-diphenyl carbazide; EDTA = ethylenediamine tetraacetate; Hepes $=\mathrm{N}$-2-(hydroxyethyl)-piperazine-N'-2ethanesulphonic acid; LHC = light-harvesting complex; Mes = 2-(N-morpholino)-ethanesulphonic acid; PS = photosystem; SDS $=$ sodium dodecyl sulphate; Tricine $=\mathrm{N}$-(tris-(hydroxymethyl)-methyl)-glycine.
} 
ATP required for $\mathrm{CO}_{2}$ fixation in bundle sheath cells by ribulose-1,5-bisphosphate carboxylase is generated by cyclic electron flow around photosystem I (PSI). Bundle sheath chloroplasts contain no PSII activity $(10,12)$.

In addition, the differentiation of maize leaves into mesophyll and bundle sheath cells makes this plant an attractive system for the study of gene expression and photosynthesis, if material of sufficient quantity and purity can be obtained. In this paper, the properties of bundle sheath and mesophyll thylakoids are described, with particular reference to the polypeptides of the light-harvesting complex of PSII (LHCII).

\section{MATERIALS AND METHODS}

\subsection{Plant Material}

Plants of Zea mays L. (cv. Dekalb DF28) were grown either for 21 days at $20{ }^{\circ} \mathrm{C}$ with a $16 \mathrm{~h}$ photoperiod at $75 \mu \mathrm{E} \cdot \mathrm{m}^{-2} \cdot \mathrm{s}^{-1}$, or for 14 days under a $12 \mathrm{~h}$ day at $28^{\circ} \mathrm{C}$ and $12 \mathrm{~h}$ night at 20 ${ }^{\circ} \mathrm{C}$, with a light intensity of $300 \mu \mathrm{E} \cdot \mathrm{m}^{-2} \cdot \mathrm{s}^{-1}$. Plants grown under these two different conditions reached a similar developmental stage. The distal halves of the second and third leaves were harvested, deribbed and homogenised in 3 volumes of ice-cold $0.4 \mathrm{M}$-sorbitol, $0.1 \mathrm{M}$ Tricine, pH 7.8, $10 \mathrm{~mm}-\mathrm{NaCl}, 5 \mathrm{~mm}-\mathrm{MgCl}_{2}$, using two $5 \mathrm{sec}$ bursts of a modified Braun homogeniser fitted with replaceable injector blades (8). The resulting slurry was squeezed through one layer of $20 \mu \mathrm{m}$ nylon mesh and allowed to pass through a second layer without squeezing. The filtrate contained very pure mesophyll chloroplasts and thylakoids.

\subsection{Isolation of bundle sheath thylakoids}

The debris retained by the first nylon mesh was resuspended in fresh grinding medium and passed through $1.67 \mathrm{~mm}$ stainless steel mesh to remove large pieces of leaf. The filtrate was concentrated by squeezing through $20 \mu$ m nylon mesh and the residue resuspended in $0.6 \mathrm{M}$-sorbitol, 20 mM-Mes, pH 5.5, 50 mm-ascorbate, 5 $\mathrm{mM}-\mathrm{MgCl}_{2}, 0.2 \%$ bovine serum albumin, containing $2 \%$ cellulase and $0.1 \%$ Macerozyme (both Onozuka R-10 from Yakult Biochemicals). The resulting dilute suspension $(500 \mathrm{ml}$ for each $50 \mathrm{~g}$ of deribbed leaves) was incubated for $3 \mathrm{~h}$ at $30^{\circ} \mathrm{C}$ with gentle agitation in a 2 litre beaker in the dark. It was then carefully poured through $0.5 \mathrm{~mm}$ stainless steel mesh and the filtrate passed through $80 \mu \mathrm{m}$ nylon mesh. Mesophyll protoplasts passed through the 80 $\mu \mathrm{m}$ nylon mesh, while the purified bundle sheath strands which were retained by this filter were extensively washed with $0.6 \mathrm{M}$-sorbitol, 20 mM-Mes, $\mathrm{pH} 7.6,0.2 \%$ bovine serum albumin. This material, designated BSI, was extremely pure, but was obtained in low yield. Most of the bundle sheath strands were retained on the 0.5 $\mathrm{mm}$ mesh and these were washed as for BSI, and vortexed in aliquots to disrupt any remaining, partially digested mesophyll cells. The pooled suspensions were passed through $80 \mu \mathrm{m}$ nylon mesh and the material retained, designated BSII, consisted of large quantities of bundle sheath strands, slightly less pure than BSI.

The BSII preparation was resuspended in 0.4 M-sorbitol, $25 \mathrm{~mm}$-Hepes, $10 \mathrm{~mm}-\mathrm{NaCl}, 5 \mathrm{~mm}$ $\mathrm{MgCl}_{2}$ and ground extensively in a mortar and pestle in the presence of small glass beads. After centrifugation for $10 \mathrm{~min}$ at $1400 \times \mathrm{g}$, the thylakoid pellet was twice resuspended in $25 \mathrm{~mm}$ Hepes, pH 7.5, 10 mM-EDTA and centrifuged for $10 \mathrm{~min}$ at $10000 \times \mathrm{g}$. The final pellet was suspended in $1.9 \mathrm{M}$-sucrose, $25 \mathrm{mM}$-Hepes, $\mathrm{pH}$ $7.5,10 \mathrm{~mm}$-EDTA and purified by flotation as previously described (3). The BSI strands were treated in a similar way, except that grinding was done in a Potter-Elvehjem mortar without glass beads, and the suspension filtered through 20 $\mu \mathrm{m}$ nylon mesh and centrifuged for $10 \mathrm{~min}$ at $1400 \times \mathrm{g}$. The thylakoid pellet was washed twice, as for BSII and purified by flotation.

\subsection{Other}

Non-denaturing and SDS-urea polyacrylamide gel electrophoresis were performed as described in (4). PSII electron transport measurements were made with an Aminco DW-2a spectrophotometer using DPC and DCPIP as electron donor and acceptor (2). Solid dilution, low temperature fluorescence emission spectra (15) were made using the fibre optics system described in (14). Thin section electron mi- 
Table I. Properties of Zea mays thylakoids

\begin{tabular}{llll}
\hline Material & $\begin{array}{l}75 \mu \mathrm{E} \cdot \mathrm{m}^{-2} \cdot \mathrm{s}^{-1} \\
\mathrm{Chl} a / b\end{array}$ & $\mathrm{Chl} a / b$ & \\
\hline unfractionated & & & \\
thylakoids & 3.40 & 3.83 & - \\
bundle sheath I & 5.67 & 6.89 & 2.1 \\
bundle sheath II & 5.51 & 6.40 & 2.6 \\
mesophyll & 3.22 & 3.50 & $100^{*}$ \\
\hline
\end{tabular}

* corresponding to $198 \mu$ moles DCPIP $\cdot \mathbf{h}^{-1} \cdot \mathbf{m g}^{-1} \mathrm{Chl}$ (with DPC as donor)

croscopy was performed as in (3) and freezefracture as in (13).

\section{RESULTS AND DISCUSSION}

Large quantities (1.5 mg chlorophyll for BSI, $10 \mathrm{mg}$ chlorophyll for BSII) of purified maize bundle sheath thylakoids were isolated using the technique described under Materials and Methods. The use of replaceable razor blades $(8)$ to homogenise the leaves resulted in less damage to the bundle sheath strands and enabled the isolation of mesophyll chloroplasts which were substantially free of bundle sheath chloroplasts. Enzymatic digestion of the crude bundle sheath preparation using macerozyme and cellulase removed the mesophyll cells adhering to the bundle sheath strands. The subsequent purification of bundle sheath thylakoids by flotation in a stepwise sucrose gradient not only removed non-chloroplast contaminants, but also mesophyll thylakoids, which had a lower density.

The extent of contamination of the bundle sheath thylakoid preparations by mesophyll thylakoids was primarily assessed by PSII electron transport activity, which is found in mesophyll thylakoids, but not in bundle sheath thylakoids $(10,12)$. The degree of contamination thus determined varied between 2.1 and $2.6 \%$ (Table I). This is in good agreement with the high chlorophyll a/b ratios (5.5 to 6.9) of the different bundle sheath preparations (Table I). This is at least as good as that reported by SCHUSTER et al. (12), who used a similar method involving enzymatic digestion, and a significant improvement on the purely mechanical method used by BRoGLIE et al. (5), who report a chlorophyll $a / b$ ratio of 4.3. The chlorophyll-protein composi- tion (Figure 1) of mesophyll and bundle sheath thylakoids was also consistent with a high degree of purity, and showed the absence of the PSII-associated $\mathrm{Chl}_{\alpha}-\mathrm{P} 2$ and $\mathrm{Chl}_{\alpha}-\mathrm{P} 3$ (CP47 and CP43,

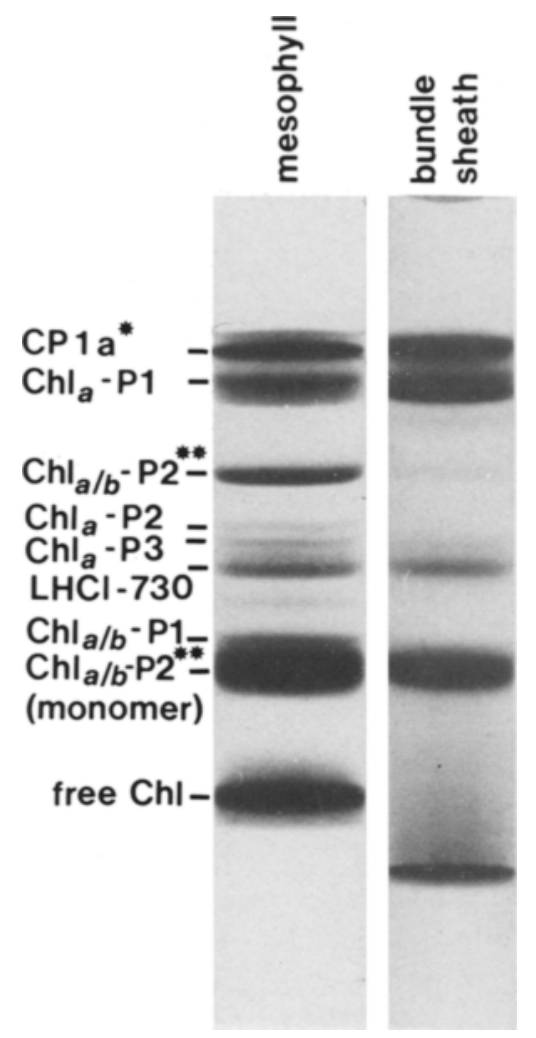

Figure 1. Chlorophyll-protein composition, by nondenaturing SDS-PAGE $\left(4^{\circ} \mathrm{C}\right)$, of octyl glucoside solubilised thylakoids purified from bundle sheath and mesophyll cells of maize. $\mathrm{Chl}_{a}-\mathrm{P} 2, \mathrm{Chl}_{a}-\mathrm{P} 3$ and $\mathrm{Chl}_{a, b}-$ $\mathrm{Pl}$ are not present in bundle sheath thylakoids, consistent with their exclusive localisation in PSII in grana. $\mathrm{Chl}_{a / p}-\mathrm{P} 2^{* *}$ is present mainly in its monomeric form and acts as a PSI light-harvesting antenna. 


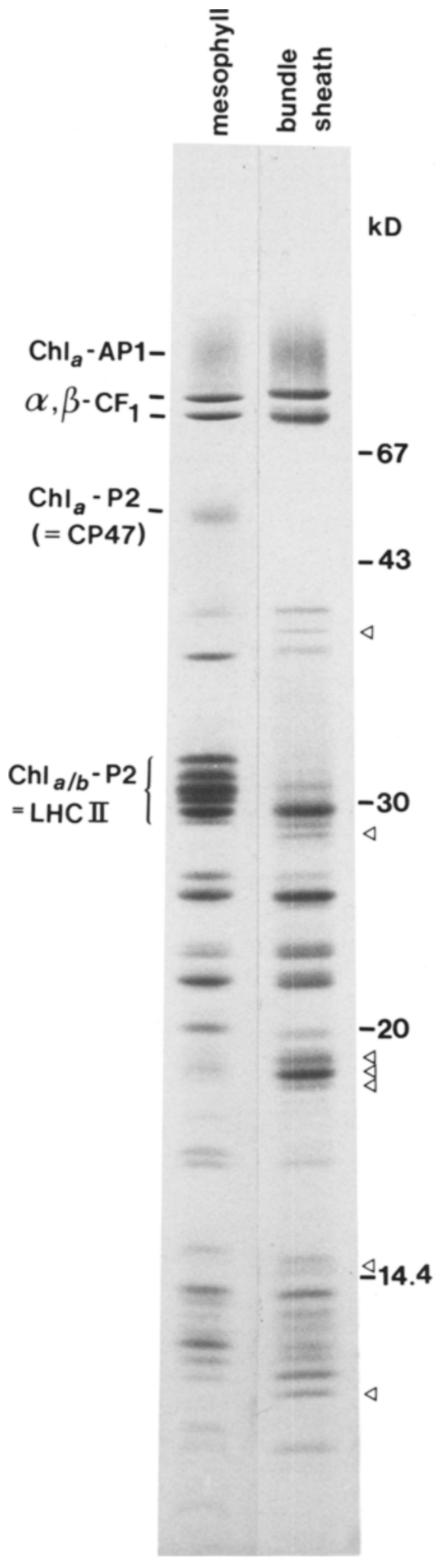

respectively). While $\mathrm{Chl}_{a / b}-\mathrm{Pl}$ was also absent, the level of $\mathrm{Ch}_{\alpha / b}-\mathrm{P} 2^{* *}$ (LHCII) was much higher than could be accounted for by a $2-3 \%$ contamination by mesophyll thylakoids (Figure 1). Higher light intensity during growth has no effect on mesophyll thylakoid composition, but there was less $\mathrm{Chl}_{\alpha / b}-\mathrm{P} 2^{* *}$ in bundle sheath thylakoids, and it was present in the monomeric form. The chlorophyll $a / b$ ratio of isolated bundle sheath thylakoids is thus a function of light intensity during growth, as well as their purity.

The differences between mesophyll and bundle sheath thylakoids were confirmed and extended by the results of polypeptide analysis (Figure 2). Of particular interest is the composition of the LHCII region, which consists of 5 polypeptides in mesophyll thylakoids. In bundle sheath thylakoids, there were only 2 polypeptides in this region. The identity of these polypeptide bands was confirmed by isolation of pure LHCII from bundle sheath and mesophyll thylakoids using Triton X-100 solubilisation and $\mathrm{Mg}^{++}$precipitation (6). This was possible because of the relatively large yield of purified bundle sheath thylakoids obtained $(1.5 \mathrm{mg}$ chlorophyll for BSI and $10 \mathrm{mg}$ chlorophyll for BSIl from $50 \mathrm{~g}$ of starting material, compared with only $0.3 \mathrm{mg}$ chlorophyll of bundle sheath thylakoids from $4 \mathrm{~g}$ of hand sliced leaves (3)). The five polypeptides of LHCII isolated from mesophyll cells had mobilities corresponding to $30,29.5,28.8,28.5$ and $26 \mathrm{kD}$, while those of LHCII from bundle sheath thylakoids had mobilties corresponding to 28 and $27.5 \mathrm{kD}$ (Figure 3). Although bundle sheath thylakoids have an active protein kinase (12), we were not able to phosphorylate either of the bundle sheath

Figure 2. Polypeptide composition of thylakoids from mesophyll and bundle sheath cells of maize using SDS-PAGE ( 10 to $20 \%$ acrylamide gradient) in the presence of $6 \mathrm{M}$-urea. Several differences are evident in the position and relative intensity of the Coomassiestained bands. Note particularly the absence of the 43-51 kD polypeptides of the PSII reaction centre and the different composition of the LHCII apoproteins. Bands marked with an arrowhead are those found in bundle sheath but not mesophyll thylakoids. 
LHCII polypeptides with ${ }^{32} \mathrm{P}$-labelled $\gamma$-ATP in vitro under conditions which labelled LHCII of mesophyll thylakoids. Polypeptides present in bundle sheath thylakoids, but not mesophyll thylakoids are indicated by arrowheads in Figure 2.

LHCII isolated from purified mesophyll and bundle sheath thylakoids was examined by freeze-fracture electron microscopy. In both cases, circular shaped freeze-fracture particles were present in densely packed regions (Figures 4 and 5), resembling those seen in LHCII isolated from barley (13). However, while the freezefracture particles of mesophyll cell LHCII were aligned in regular arrays, those of bundle sheath LHCII were not organised into arrays. Moreover, messophyll thylakoid LHCII spontaneously formed vesicles, with areas of tight adhesion (as found for barley (13)), while bundle sheath LHCII was organised into large sheets which, although aligned parallel, were not in close contact (Figures 4 and 5). It is thus clear that LHCII from mesophyll and bundle sheath thylakoids differs not only in polypeptide composition (Figure 3), but also in ultrastructural organisation.
The low temperature fluorescence emission spectra (Figure 6) show the usual three peaks at 685,695 and $735 \mathrm{~nm}$ for mesophyll thylakoids. In contrast, bundle sheath thylakoids have a single emission maximum at $732 \mathrm{~nm}$ indicating that PSII is absent and that the LHCII present is functionally attached to PSI since its fluorescence at $685 \mathrm{~nm}$ is quenched by the PSI reaction centre complex.

The presence of LHCII, but none of the PSII core polypeptides, is in contrast to the findings of SCHUSTER et al. (12), who report the presence of some of the PSII-core polypeptides, but no LHCII in bundle sheath thylakoids. We believe this could be due to these authors' use of plant material at an earlier developmental stage. This is indicated by the short incubation time (20-30 min) used for enzymatic digestion (12). Bundle sheath chloroplasts from the basal half of the leaf have numerous small grana, which contain the PSII-containing EFs particles (11). As the leaves develop, the grana and PSII activity disappear (3). This may also explain the very low levels of LHCII found in their bundle sheath thylakoids (12). In contrast, the high levels of the PSII-associated polypeptides 5 and $6\left(=\mathrm{Chl}_{a}-\mathrm{P} 2\right.$ and

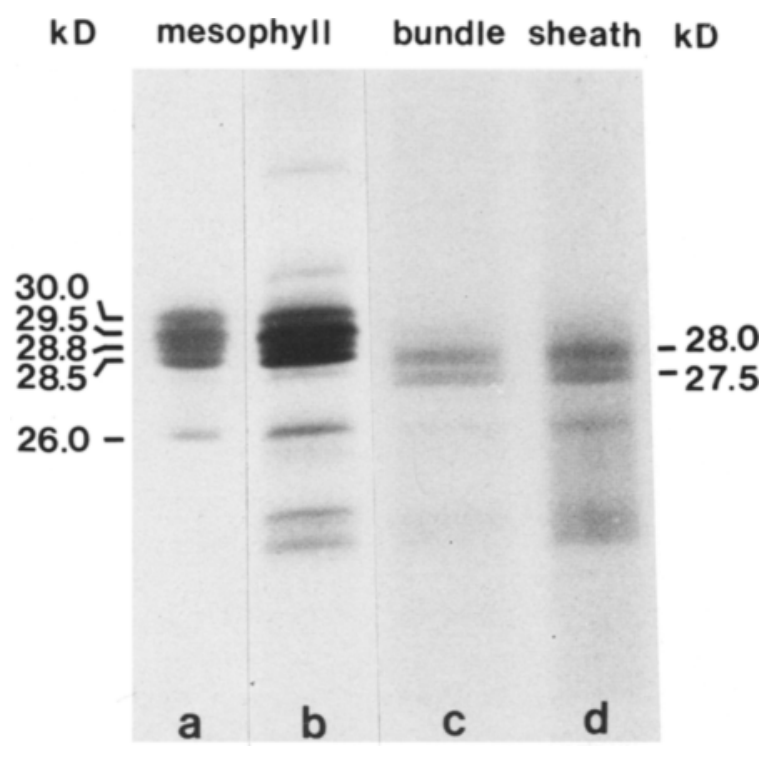

Figure 3. Polypeptide composition of LHCIl isolated from (a) purified mesophyll and (c) bundle sheath thylakoids. The polypeptide pattern from the corresponding region of thylakoids $(b, d)$ is shown for comparison. Arrows indicate the five polypeptides of mesophyll LHCII and the two of bundle sheath LHCII. 

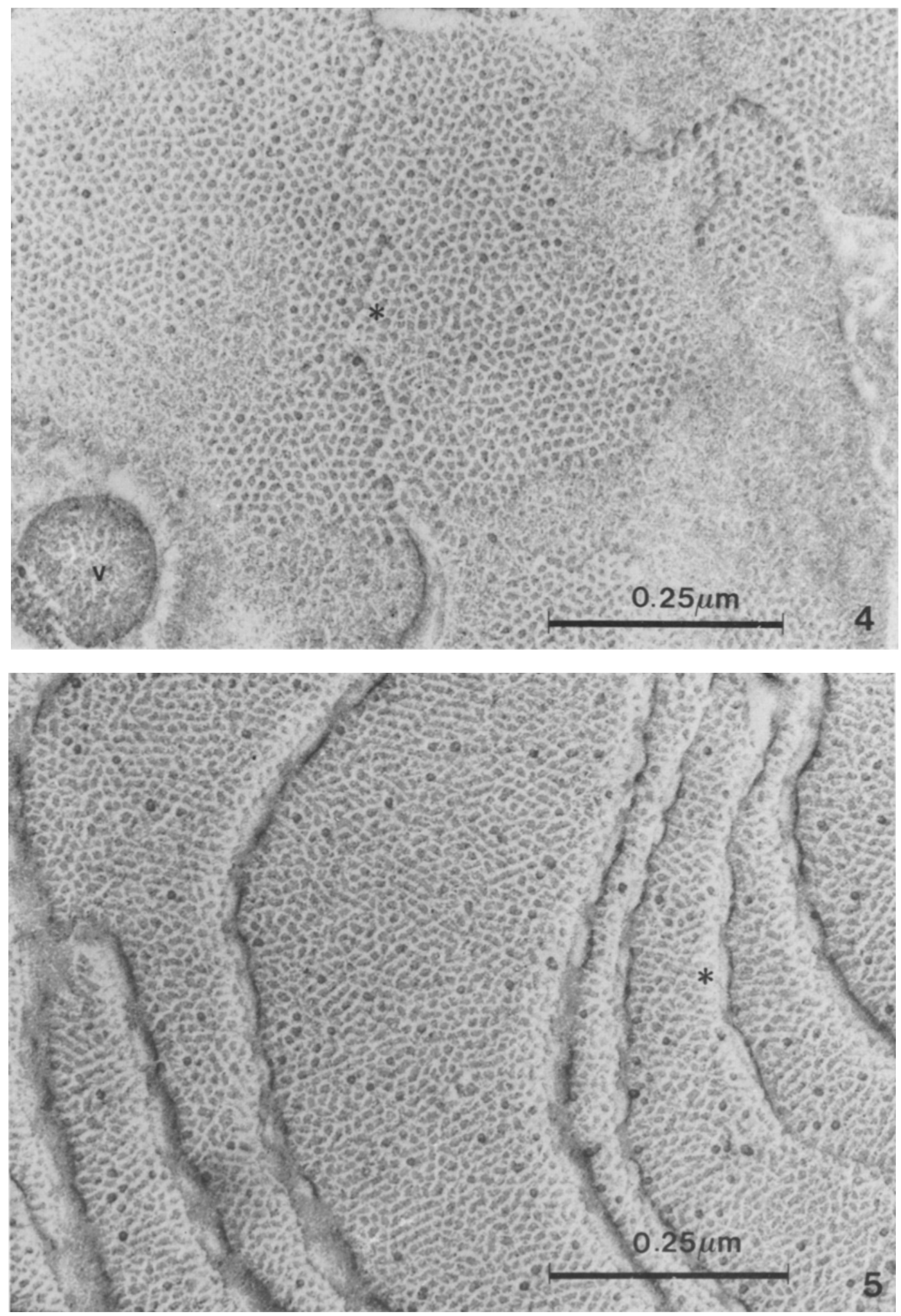


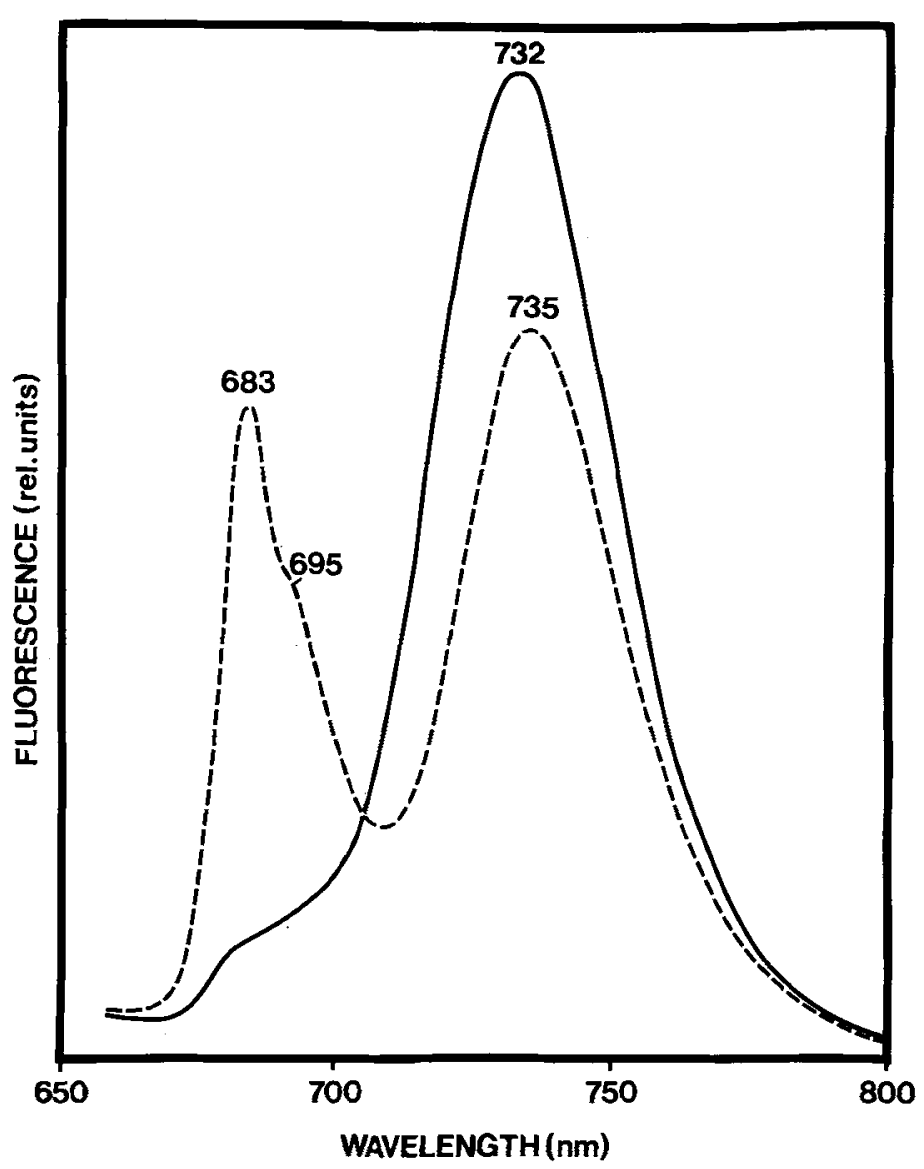

Figure 6. Low temperature fluoresence emission spectra of bundle sheath strands and mesophyll protoplasts. To eliminate artefacts due to self-absorption at short wavelengths $(<700 \mathrm{~nm})$ the solid dilution technique described by WEIS (1985) was used. The $685 \mathrm{~nm}$ peak typical of LHCII is not evident in the spectrum from bundle sheath strands, showing that the $\mathrm{LHCll}$ present is efficiently quenched by the PSI reaction centre.

$\mathrm{Chl}_{\alpha}-\mathrm{P3}$ ) in maize bundle sheath thylakoids reported by BroGLIE et al. (5), can be attributed to significant contamination of their bundle sheath preparation by mesophyll thylakoids.

It is clear, therefore, that mesophyll and bun- dle sheath thylakoids from maize differ markedly in their properties, and that the presence of LHCII in bundle sheath thylakoids cannot be explained by contamination of bundle sheath thylakoids with mesophyll thylakoids. The high

Figure 4. Freeze-fracture electron microscopy of LHCII isolated from mesophyll thylakoids of maize. Note that the approximately $75 \AA$ diameter particles are found in arrays with a spacing of about $100 \AA$. The isolated LHCII spontaneously form vesicles ( $\mathrm{v}=$ a small vesicle), which are tightly appressed (e.g., at asterisk) when $\mathrm{Mg}^{++}$is added. Magnification $\times 150,000$.

Figure 5. Freeze-fracture electron microscopy of LHCII isolated from bundle sheath thylakoids. Unlike Figure 4, the freeze-fracture particles are not organised into regular arrays, although they are densely packed. Isolated LHCII formed large sheets, rather than vesicles, and although they are aligned parallel, they do not become tightly appressed in the presence of $\mathrm{Mg}^{++}$(compare the degree of appression at the asterisk with that in Figure 4). Magnification $\times 150,000$. 
purity of the bundle sheath thylakoids is established by their low PSII-dependent electron transport activity, confirmed by their chlorophyll-protein and polypeptide composition, and their low temperature fluorescence emission spectra. Furthermore, $\mathrm{LHCll}$ isolated from the thylakoids of these two different leaf cell types have a different polypeptide composition and freeze-fracture ultrastructure. The products of the different genes of the LHCII family (7) are differentially expressed in bundle sheath and mesophyll cells. We have preliminary evidence that the polypeptide composition of LHCII in stroma and grana lamellae is also different, and we suggest that they serve different functions. Some are attached to PSI, other to PSII and a third group move from PSII to PSI after phosphorylation (9).

\section{ACKNOWLEDGEMENTS}

We wish to thank Professor DITER VON WETTSTEIN for his encouragement during the course of this work and for critically reading the manuscript. We thank NINA RASMUSSEN for drawing the figures, and ANN-SOFi STEINHOLTZ for printing the photographs.

\section{REFERENCES}

1. ANDERSSON, B. \& J M. M. ANDERSON: Lateral heterogeneity in the distribution of chlorophyll-protein complexes of the thylakoid membranes of spinach chloroplasts. Biochim. Biophys. Acta 593, 427 440 (1980)

2. BASSI, R.: Spectral properties and polypeptide composition of the chlorophyll-proteins from thylakoids of granal and agranal chloroplasts of maize (Zea mays L. ). Carlsberg Res. Commun. 50, 127-143 (1985)

3. Bassi, R. A. dal Belin-Peruffo, R. Barbato \& R. GHISI: Differences in chlorophyll-protein complexes and composition of polypeptides between thylakoids from bundle sheaths and mesophyll cells in maize. Eur. J. Biochem. 146, 589-595 (1985)

4. BASSi, R., O. Machold \& D. J. SimpSON: Chlorophyll-proteins of two photosystem I preparations from maize. Carlsberg Res. Commun. 50, 145-162 (1985)
5. Broglie, R., G. Coruzzi, B. Keith \& N. -H. Chua: Molecular biology of $\mathrm{C}_{4}$ photosynthesis in Zea mays: differential localization of proteins and mRNAs in the two leaf cell types. Plant Mol. Biol. 3, 431.444 (1984)

6. BuRKe, J. J., C. L. DitTo \& C. J. ARNTZEN: Involvement of the light-harvesting complex in cation regulation of excitation energy distribution in chloroplasts. Arch. Biochem. Biophys. 187, 252263 (1979)

7. Dunsmuir, P., S. S. Smith \& J. Bedbrook: The major chlorophyll $\mathrm{a} / \mathrm{b}$ binding protein of Petunia is composed of several polypeptides encoded by a number of distinct nuclear genes. J. Mol. Appl. Genet. 2, 285-300 (1983)

8. Kannangara, C. G., S. P. Gough, B. Hansen, J. N. RASMUSSEN \& D. J. SIMPSON: A homogeniser with replaceable razor blades for bulk isolation of active barley plastids. Carlsberg Res. Commun. 42, 431439 (1977)

9. LARSSON, U. K.\& B. ANDERSSON: Different degrees of phosphorylation and lateral mobility of two polypeptides belonging to the light-harvesting harvesting complex of Photosystem II. Biochim. Biophys. Acta 809, 396-402 (1985)

10. Leegood, R. C., D. Crowther, D. A. Walker \& G. HIND: Energetics of photosynthesis in Zea mays 1 . Studies of the flash-induced electrochromic shift and fluorescence induction in bundle sheath cells. Biochim. Biophys. Acta 722,116-126 (1983)

11. Miller, K. R., G. J. Miller \& K R. MCIntyre: Organization of the photosynthetic membrane in maize mesophyll and bundle sheath chloroplasts. Biochim. Biophys. Acta 459, 145-156 (1977)

12. Schuster, G., I. Ohad, B. Martineau \& W. C. TAYLOR: Differentiation and development of bundle sheath and mesophyll thylakoids in maize. Thylakoid polypeptide composition, phosphorylation, and organisation of photosystem II. J. Biol. Chem. 260, 11866-11873 (1985)

13. SIMPSON, D. J.: Freeze-fracture studies on barley plastid membranes III. Location of the light-harvesting chlorophyll-protein. Carlsberg Res. Commun. 44, 305-336 (1979)

14. SimPSON. D. J.\&D. von WETTSTEIN: Macromolecular physiology of plastids XIV. Viridis mutants in barley: genetic, fluoroscopic and ultrastructural characterisation. Carlsberg Res. Commun. 45, 283-314 (1980)

15. WEIS. E.: Chlorophyll fluorescence at $77 \mathrm{~K}$ in intact leaves: Characterisation of a technique to eliminate artefacts related to self-absorption. Photosyn, Res. 6, 73-86 (1985) 